\title{
A Patient With Sporotrichosis Diagnosed By Molecular Biology Combined With Traditional Methods
}

\author{
Runchao Wang ${ }^{\mathrm{a}, \mathrm{b}}$, Haixia Jing ${ }^{\mathrm{a}}$, Shaoxiu Chen ${ }^{\mathrm{a}}$, Yao Yang ${ }^{\mathrm{a}}$, \\ Hualiang Nan ${ }^{\mathrm{a}}$, Tingting Chen ${ }^{\mathrm{a}}$
}

\begin{abstract}
We present a case of sporotrichosis diagnosed by molecular biology combined with traditional methods. Fungal DNA was extracted from skin tissues of the patient by fungal DNA extraction kit. The PCR amplified fragments were compared on GenBank website, and the pathogenic fungi were identified. This case was diagnosed by molecular biology, clinical manifestations, fungal morphology and pathology. The pathogenic fungi of the patient was identified as Sporothrix globosa by comparison, combined with typical clinical manifestations, typical filamentous colonies by fungi culture and pathological findings of infectious granuloma. The case could be diagnosed as Sporothrix globosa-induced sporotrichosis. Molecular biology combined with traditional detection methods for diagnosis of sporotrichosis is helpful to improve the accuracy of diagnosis.
\end{abstract}

Keywords: Sporotrichosis; Sporothrix globosa; Molecular biology

\section{Introduction}

Sporotrichosis is a subacute or chronic fungal infection caused by the Sporothrix species. Sporothrix species can easily invade skin, subcutaneous tissue and lymphatic vessels nearby. It may cause disseminated infection in immunodeficient individuals [1]. The pathogen of the disease was previously considered to be Sporothrix schenckii, but with the development of molecular biology, it was found that the pathogen contained several fungi, called "Sporothrix schenckii complex", which included Sporothrix schenckii, Sporothrix globosa, Sporothrix albicans, and so on. The source of infection, mode of transmission, distribution pattern, virulence and reaction to antifungal drugs of various sporotrichosis are different $[2,3]$. Therefore, it is necessary to identify the pathogenic strains of sporotrichosis by

Manuscript submitted August 29, 2019, accepted September 16, 2019

aDepartment of Dematology, Taihe Hospital, Hubei University of Medicine, Shiyan 442000, China

${ }^{b}$ Corresponding Author: Runchao Wang, Department of Dematology, Taihe Hospital, Hubei University of Medicine, Shiyan 442000, China.

Email:wrczzl@163.com

doi: https://doi.org/10.14740/jmc3362 molecular biological methods. A case of sporotrichosis caused by Sporothrix globosa was reported as follows.

\section{Case Report}

\section{Clinical data}

A 57-year-old female farmer from the city of Danjiangkou, Hubei Province, was admitted to the hospital due to nodules of the left upper extremity accompanied by erosion and exudation for a month. After the injury of the index fingertip of the left hand in farming a month ago, papules and nodules of broad bean size appeared gradually in the subcutaneous area of the left forearm, accompanied by mild itching and pain. The subcutaneous nodules gradually increased and skin surface ulceration and erosion appeared. Later, similar skin lesions gradually increased and arranged along the long axis of the upper limb, showing segmental distribution. She denied special medical history. Physical examination showed a small piece of ulceration and scab at the tip of the left index finger, and the skin around it was dry and scaly (Fig. 1a). Several purple-red nodules of pigeon egg size arranged along the long axis can be seen on the left upper limb, with dark red erosive surface and a little bloody and purulent secretions (Fig. 1b). On auxiliary examination, there were no obvious abnormalities in routine blood and urine, liver and kidney function, blood sugar and lipid.

\section{Microscopic examination and culture of fungi}

The skin debris and pus of the patient was taken and placed on slide. No hyphae and/or spores were detected under microscope after adding $10 \% \mathrm{KOH}$ solution. Colonies were found after 10 days of culture in the Sabouraud medium (Fig. 2a). The morphology of hyphae and spores was observed by fluorescence staining through a microscope (Fig. 2b).

\section{Pathological examination}

Paraffin section, hematoxylin and eosin (H\&E) staining and periodic acid-Schiff (PAS) staining were performed on the biopsy tissues. H\&E staining showed infectious granuloma changes (Fig. 3a). PAS staining showed a red-stained fungal 

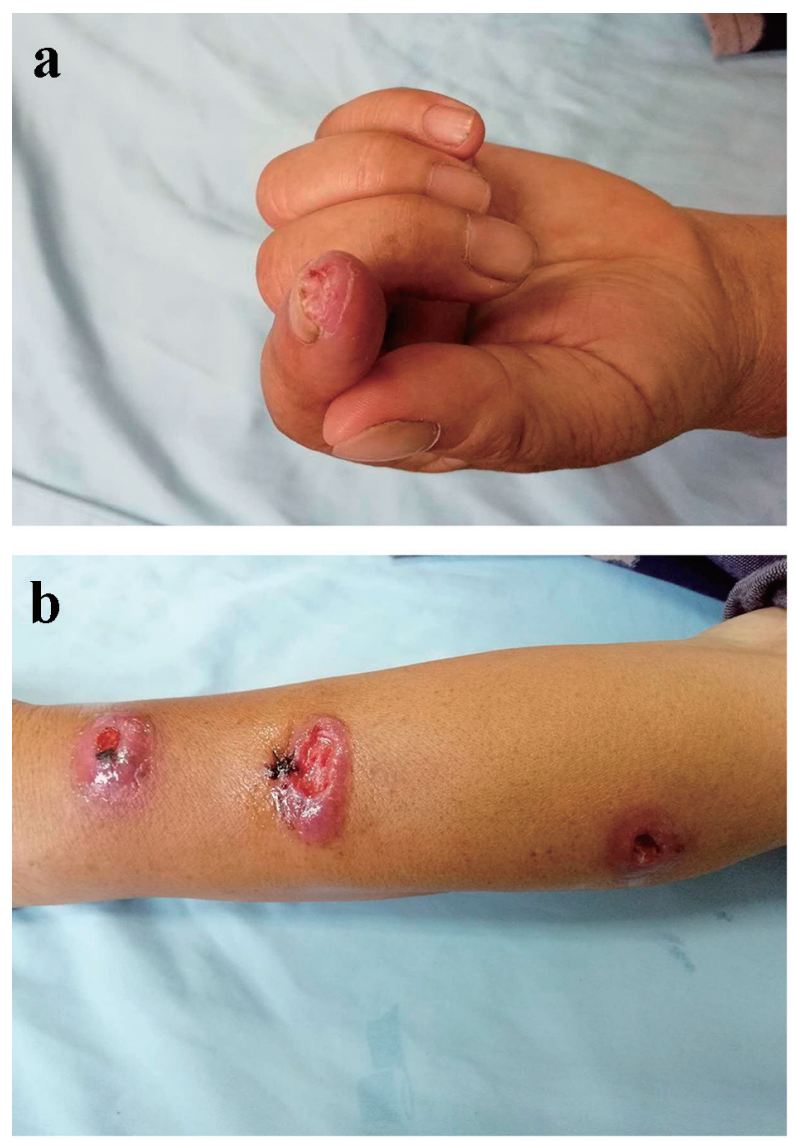

Figure 1. (a) The appearance of skin lesion at the tip of the left index finger. (b) The appearance of skin lesion on the left forearm.

spore in the deep dermis (Fig. 3b).

\section{Molecular biology assay}

The homogenate supernatant of biopsy tissues and the strains cultured were used to extract DNA by Biospin Fungal Genome Extraction Kit (BSC14S1, Hangzhou Bori Technology Co., Ltd). Specific methods were referred to the instructions of the kit. The amplification primers CL1 and CL2A were used in the PCR reaction [4]. The amplification method of PCR was detailed in Reference [5]. For each reaction, we added 20 - 60 ng of DNA template and a $0.5-1 \mathrm{mM}$ concentration of each primer in a total volume of $25 \mu \mathrm{L}$. The amplification program included an initial denaturation at $94{ }^{\circ} \mathrm{C}$ for $5 \mathrm{~min}$, followed by 35 cycles consisting of denaturation at $95^{\circ} \mathrm{C}$ for $30 \mathrm{~s}$, annealing for $1 \mathrm{~min}$ at $60{ }^{\circ} \mathrm{C}$ and extension for $1 \mathrm{~min}$ at $72{ }^{\circ} \mathrm{C}$. A final extension step of $72{ }^{\circ} \mathrm{C}$ for 7 min was included. After the reaction, PCR products were sequenced by Sangon Biotech (Shanghai) Co., Ltd. The sequences thus obtained were compared on GenBank website (http://www.ncbi.nlm.nih.gov/ blast). The DNA amplification products extracted from both the homogenate supernatant of biopsy tissue and the cultured strains were identified as Sporothrix globosa.

\section{Diagnosis and treatment}

According to the clinical manifestations, fungal culture, pathological examination and molecular biology identification results, the patient could be diagnosed as Sporothrix globosa-induced sporotrichosis. According to the morphology of lesions, the patient was diagnosed as lymphatic type. Itraconazole capsules were given $100 \mathrm{mg}$ per time, twice a day. The patient was discharged before the skin lesion was significantly improved due to the limited economic conditions. The patient was followed up by telephone outside hospital. She continued to take medication to consolidate her treatment. So far, the effect was obvious, and there was no recurrence.

\section{Discussion}

Sporotrichosis is a common deep fungal dermatosis in China. The main pathogen is Sporothrix globosa [6]. Farmers, gar-
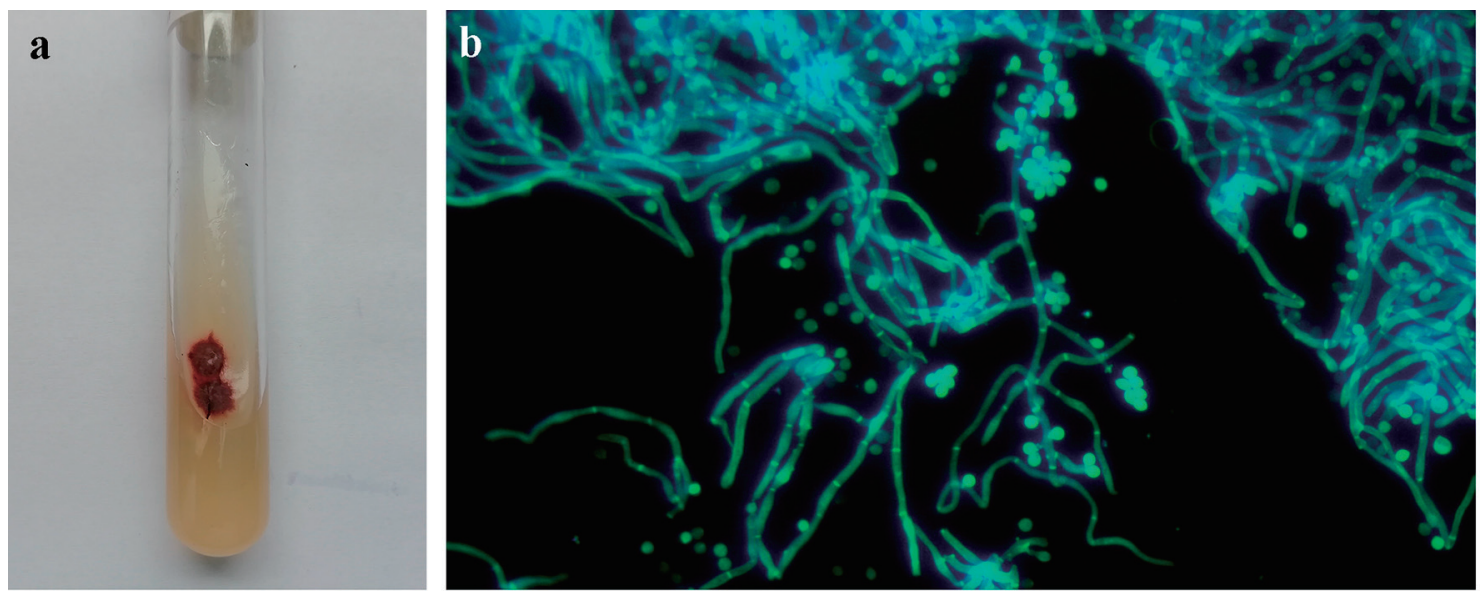

Figure 2. (a) The appearance of colonies cultured in the Sabouraud medium for 10 days. (b) The morphology of fungi from cultured colonies under the fluorescence through a microscope. 

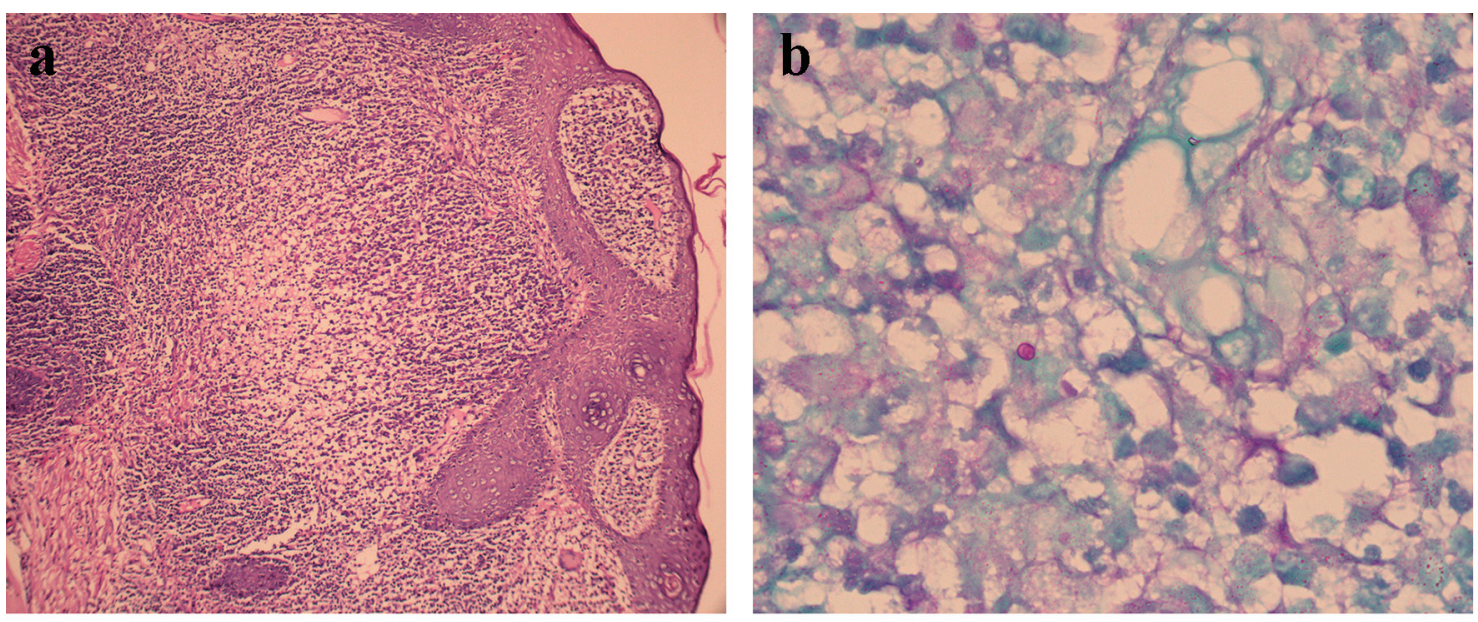

Figure 3. (a) Histopathological images of lesion tissue. Diffuse mixed inflammatory cell infiltration in the dermis, including lymphocyte, neutrophil, eosinophil and plasma cells (H\&E, $\times 100)$. (b) Histopathological images of lesion tissue and a red-stained spore in the deep dermis (PAS, $\times$ 400). H\&E: hematoxylin and eosin; PAS: periodic acid-Schiff.

deners and forestry workers engaged in field work are liable to be infected with the disease. In addition, some animals, such as cats and dogs, are also susceptible to sporotrichosis and the source of its infection [7-9]. Sporotrichosis has a clear etiology and geographical distribution. It has a high incidence in Northeast China and has been reported frequently in other areas of China [10]. In this study, the patient was farmer. She came from the local area and had not been anywhere else for half a year before the onset of the disease, indicating that Sporothrix species also existed in the local environment. The patient in this area may be infected by Sporothrix species when they were engaged in agricultural work.

When the pathogen affects the skin, it can be divided into fixed type, lymphatic type and disseminated type according to the different morphology of the skin lesion, and disseminated type is rare. The lesions may be infiltrative plaques or nodules with erosion or ulceration, accompanied by purulent exudation, and may be isolated or distributed along lymphatic vessels. In this study, the case was lymphatic type. The skin lesions were typical. The patient can be initially diagnosed as sporotrichosis. The definite diagnosis depended on laboratory examination.

At present, laboratory examinations that commonly used include fungal microscopy, culture and histopathological examination. The positive rate of direct microscopic examination of fungi is low, culture usually takes $1-2$ weeks, and histopathological examination is not easy to find characteristic fungal spores. Therefore, it brings some difficulties to clinical diagnosis and treatment. Molecular biology examination is time saving and accurate, which is conducive to early diagnosis and accurate diagnosis, but it requires certain equipment and consumables, which is not suitable for the primary hospitals. At present, positive fungal culture is still the gold standard for diagnosis of sporotrichosis. However, with the continuous development of science and basic hospital facilities, molecular biology technology will be more clinical and grass-roots. In this study, fungal DNA extracted from the homogenate supernatant of lesion tissue of the patient was identical with that extracted from fungal culture. The results showed that fungal dermatosis could be rapidly and accurately diagnosed by molecular biology detection in clinical practice. The combination of traditional detection methods and molecular biology detection was beneficial to avoid errors and improve the detection efficiency. The accuracy of diagnosis provides a solid foundation for further treatment and epidemiology research.

Potassium iodide is used to be the first choice for the treatment of sporotrichosis, with definite curative effect. However, due to its inconvenient to use and some side effects (rash, gastrointestinal irritation, arthralgia, thyroid suppression, etc.), its further application is limited [11]. At present, itraconazole or terbinafine are used as the first-line drug, which are safer and more efficient [12]. In our study, the patient was treated with itraconazole capsule $200 \mathrm{mg}$ /day and she improved markedly without recurrence.

\section{Acknowledgments}

We are grateful to the medical staff who have treated this patient carefully.

\section{Funding Disclosure}

This is a clinical observation article without any funding.

\section{Conflict of Interest}

The authors declare that they have no conflict of interest.

\section{Informed Consent}

We got informed consent from patient. 


\section{Author Contributions}

Runchao Wang summarized and wrote the article. Shaoxiu Chen, Hualiang Nan and Yao Yang collected the case data. Tingting Chen and Haixia Jing wrote the article.

\section{Reference}

1. Orofino-Costa R, Macedo PM, Rodrigues AM, Bernardes-Engemann AR. Sporotrichosis: an update on epidemiology, etiopathogenesis, laboratory and clinical therapeutics. An Bras Dermatol. 2017;92(5):606-620.

2. Lopes-Bezerra LM, Mora-Montes HM, Zhang Y, Nino-Vega G, Rodrigues AM, de Camargo ZP, de Hoog S. Sporotrichosis between 1898 and 2017: The evolution of knowledge on a changeable disease and on emerging etiological agents. Med Mycol. 2018;56(suppl_1):126-143.

3. Bonifaz A, Tirado-Sanchez A. Cutaneous Disseminated and Extracutaneous Sporotrichosis: Current Status of a Complex Disease. J Fungi (Basel). 2017;3(1):6.

4. O'Donnell K, Nirenberg HI, Aoki T, et al. A multigene phylogeny of the Gibberella fujikuroi species complex: detection of additional phylogenetically distinct species. Mycoscience. 2000;41(1):61-78.

5. Marimon R, Gene J, Cano J, Trilles L, Dos Santos Laz- era M, Guarro J. Molecular phylogeny of Sporothrix schenckii. J Clin Microbiol. 2006;44(9):3251-3256.

6. Chen M, Xu Y, Hong N, Yang Y, Lei W, Du L, Zhao J, et al. Epidemiology of fungal infections in China. Front Med. 2018;12(1):58-75.

7. Gremiao ID, Miranda LH, Reis EG, Rodrigues AM, Pereira SA. Zoonotic epidemic of sporotrichosis: cat to human transmission. PLoS Pathog. 2017;13(1):e1006077.

8. Barros MB, Schubach Ade O, do Valle AC, Gutierrez Galhardo MC, Conceicao-Silva F, Schubach TM, Reis $\mathrm{RS}$, et al. Cat-transmitted sporotrichosis epidemic in Rio de Janeiro, Brazil: description of a series of cases. Clin Infect Dis. 2004;38(4):529-535.

9. Schubach TM, de Oliveira Schubach A, dos Reis RS, Cuzzi-Maya T, Blanco TC, Monteiro DF, Barros BM, et al. Sporothrix schenckii isolated from domestic cats with and without sporotrichosis in Rio de Janeiro, Brazil. Mycopathologia. 2002;153(2):83-86.

10. Chakrabarti A, Bonifaz A, Gutierrez-Galhardo MC, Mochizuki T, Li S. Global epidemiology of sporotrichosis. Med Mycol. 2015;53(1):3-14.

11. Costa RO, Macedo PM, Carvalhal A, Bernardes-Engemann AR. Use of potassium iodide in dermatology: updates on an old drug. An Bras Dermatol. 2013;88(3):396402.

12. Mahajan VK. Sporotrichosis: an overview and therapeutic options. Dermatol Res Pract. 2014;2014:272376. 